\title{
Explicit computation of the variance of the number of maxima in hypercubes
}

\section{Christian Costermans and Hoang Ngoc Minh}

Université Lille II, 1, Place Déliot, 59024 Lille, France

We present a combinatorial approach of the variance for the number of maxima in hypercubes. This leads to an explicit expression, in terms of Multiple Zeta Values, of the dominant term in the asymptotic expansion of this variance. Moreover, we get an algorithm to compute this expansion, and show that all coefficients occuring belong to the $\mathbb{Q}$ algebra generated by Multiple Zeta Values, and by Euler's constant $\gamma$.

Keywords: Maximal points, harmonic sums, shuffle algebra, multiple zeta values

\section{Introduction}

Let $\Lambda=\left\{x_{1}, \ldots, x_{n}\right\}$ be a set of independent and identically distributed random vectors in $\mathbb{R}^{d}$. A point $x_{i}=\left(x_{i_{1}}, \ldots, x_{i_{d}}\right)$ is said to be dominated by $x_{j}=\left(x_{j_{1}}, \ldots, x_{j_{d}}\right)$ if $x_{i_{k}}<x_{j_{k}}$ for all $k \in\{1, \ldots, d\}$ and a point $x_{i}$ is called a maximum of $\Lambda$ if none of the other points dominates it. The number of maxima of $\Lambda$ is denoted by $K_{n, d}$.

Many papers were already devoted to the study of the number of maxima in a set of points, since it arises in various domains. Recently, in [2], Bai et al. proposed a method for computing an asymptotic expansion of the variance.

The study of $\operatorname{Var}\left(K_{n, d}\right)$ for random samples from $[0,1]^{d}$ is precisely the goal of the present paper. For that, we exploit an important result, first derived by Ivanin [5] :

$$
\mathbb{E}\left(K_{n, d}^{2}\right)=\mu_{n, d}+\sum_{1 \leq t \leq d-1}\left(\begin{array}{l}
d \\
t
\end{array}\right) \sum_{l=1}^{n-1} \frac{1}{l} \sum^{(*)} \frac{1}{i_{1} \ldots i_{d-2} j_{1} \ldots j_{d-1}},
$$

where the $\operatorname{sum}(*)$ is taken over indices verifying

$$
1 \leq i_{1} \ldots \leq i_{t-1} \leq l, 1 \leq i_{t} \leq \ldots \leq i_{d-2} \leq l \quad \text { and } \quad l+1 \leq j_{1} \leq \ldots \leq j_{d-1} \leq n .
$$

In Formula [1], $\mu_{n, d}$ stands for the mean of $K_{n, d}$, first calculated by Barndorff-Nielsen and Sobel [3] :

$$
\mu_{n, d}=\mathbb{E}\left(K_{n, d}\right)=\sum_{1 \leq i_{1} \leq \ldots \leq i_{d-1} \leq n} \frac{1}{i_{1} \ldots i_{d-1}} .
$$

After having given an alternative derivation for this formula, Bai et al. deduce, by analytic and combinatoric considerations, as the main result of [1], the following equivalent

$$
\begin{array}{r}
\operatorname{Var}\left(K_{n, d}\right) \sim\left(\frac{1}{(d-1) !}+\kappa_{d}\right) \ln ^{d-1}(n), \\
\text { with } \kappa_{d}=\sum_{t=1}^{d-2} \frac{1}{t !(d-1-t) !} \sum_{l \geq 1} \frac{1}{l^{2}} \sum^{(* *)} \frac{1}{i_{1} \ldots i_{t-1} j_{1} \ldots j_{d-2-t}}
\end{array}
$$

the sum $(* *)$ being calculated over all indices verifying $1 \leq i_{1} \leq \ldots \leq i_{t-1} \leq l$ and $1 \leq j_{1} \leq \ldots \leq$ $j_{d-2-t} \leq l$.

These two formulas give rise to harmonic sums $\mathrm{A}_{\mathbf{s}}(N)$, closely related to $\mathrm{H}_{\mathbf{s}}(N)$ defined for a multiindex $\mathbf{s}=\left(s_{1}, \ldots, s_{r}\right)$ by

$$
\mathrm{A}_{\mathbf{s}}(N)=\sum_{N \geq n_{1} \geq \ldots \geq n_{r} \geq 1} \frac{1}{n_{1}^{s_{1}} \ldots n_{r}^{s_{r}}}, \quad \mathrm{H}_{\mathbf{s}}(N)=\sum_{N \geq n_{1}>\ldots>n_{r} \geq 1} \frac{1}{n_{1}^{s_{1}} \ldots n_{r}^{s_{r}}} .
$$

1365-8050 (C) 2006 Discrete Mathematics and Theoretical Computer Science (DMTCS), Nancy, France 
We already studied in [4] the algebraic properties of $\mathrm{H}_{\mathbf{s}}(N)$; in particular, when $s_{1}>1, \mathrm{H}_{\mathbf{s}}(N)$ converges to the polyzêta (or MZV) $\zeta(\mathbf{s})=\sum_{n_{1}>\ldots>n_{r} \geq 1} \frac{1}{n_{1}^{s_{1}} \ldots n_{r}^{s_{r}}}$. There exist explicit relations, given by Hoffman between the $\mathrm{A}_{\mathbf{s}}(N)$ and $\mathrm{H}_{\mathbf{s}}(N)$. Indeed, let $\operatorname{Comp}(n)$ be the set of compositions of $n$, i.e. sequences $\left(i_{1}, \ldots, i_{r}\right)$ of positive integers summing to $n$. If $I=\left(i_{1}, \ldots, i_{r}\right)$ (resp. $J=\left(j_{1}, \ldots, j_{p}\right)$ ) is a composition of $n$ (resp. of $r$ ) then $J \circ I=\left(i_{1}+\ldots+i_{j_{1}}, i_{j_{1}+1}+\ldots+i_{j_{1}+j_{2}}, \ldots, i_{k-j_{p}+1}+\ldots+i_{k}\right)$ is a composition of $n$. One has $(l(J)$ being the number of parts of $J)$ :

$$
\mathrm{A}_{\mathbf{s}}(N)=\sum_{J \in \operatorname{Comp}(r)} \mathrm{H}_{J \circ \mathbf{s}}(N) \quad \text { and } \quad \mathrm{H}_{\mathbf{s}}(N)=\sum_{J \in \operatorname{Comp}(r)}(-1)^{l(J)-r} \mathrm{~A}_{J \circ \mathbf{s}}(N) .
$$

Here, the nature of Formulas (2) and (4) makes clear that it would be difficult to interpret both formulas in terms of $\mathrm{H}_{\mathbf{s}}(N)$. So, we prefer looking at the algebraic and combinatoric properties of $\mathrm{A}_{\mathbf{s}}(N)$, and deduce from these ones two main results, first the explicit value of $\kappa_{d}$ in terms of Multiple Zeta Values, and then an algorithm to compute the asymptotic expansion of $\operatorname{Var}\left(K_{n, d}\right)$.

\section{Combinatoric background}

\subsection{Combinatorics on words}

To the multi-index $\mathbf{s}=\left(s_{1}, \ldots, s_{r}\right)$ we can canonically associate the word $v=y_{s_{1}} \ldots y_{s_{r}}$ over the infinite alphabet $Y=\left\{y_{i}\right\}_{i \geq 1}$. Its length $r$ is denoted by $\ell(v)$, and its weight is defined as $|v|=\sum_{i=1}^{r} s_{i}$. The number of occurences of the letter $y_{i}$ in the word $w$ is denoted by $N_{i}(w)$. Moreover, the empty multi-index will correspond to the empty word $\epsilon$.

Example 1 Let $w=y_{1} y_{4} y_{1}^{2} y_{2}$, we have $\ell(w)=5,|w|=9$ and $N_{1}(w)=3$.

Definition 1 Let $S$ be a subset of $Y$, and $\rho$ a positive integer, we define $S_{\rho}$ as the set of words containing only letters in $S$, and of weight equal to $\rho$.

Example 2 Let $S=\left\{y_{1}, y_{2}\right\}$ and $\rho=4$ then $S_{\rho}=\left\{y_{1}^{4}, y_{1} y_{2} y_{1}, y_{1}^{2} y_{2}, y_{2} y_{1}^{2}, y_{2}^{2}\right\}$.

We shall henceforth identify the multi-index $\mathbf{s}$ with its encoding by the word $v=y_{s_{1}} \ldots y_{s_{r}}$.

We denote by $Y^{*}$ the free monoid generated by $Y$, which is the set of words over $Y$, and by $\mathbb{Q}\langle Y\rangle$ the algebra of non commutative polynomials with coefficients in $\mathbb{Q}$.

\subsection{Shuffle product}

Let $y_{i}, y_{j} \in Y$ and $u, v \in Y^{*}$. The minus-stuffle ${ }^{(\mathrm{i})}$ of $u=y_{i} u^{\prime}$ and $v=y_{j} v^{\prime}$ is the polynomial recursively defined by

$$
\begin{aligned}
& \epsilon \sqcup u=u \sqcup \epsilon=u \quad \text { and } \\
& u \sqcup v=y_{i}\left(u^{\prime} \sqcup v\right)+y_{j}\left(u \sqcup v^{\prime}\right)-y_{i+j}\left(u^{\prime} \sqcup v^{\prime}\right)
\end{aligned}
$$

For example, $y_{1} \hookleftarrow y_{2}=y_{1} y_{2}+y_{2} y_{1}-y_{3}$.

Proposition $1 y_{1}^{r}=\sum_{\substack{s_{1}, \ldots, s_{r}>0 \\ s_{1}+\ldots+r s_{r}=r}} \frac{\left.y_{1}^{\llcorner-1} s_{1} \sqcup\right\rfloor \sqcup y_{r}^{\sqcup-s_{r}}}{1^{s_{1}} s_{1} ! \ldots r^{s_{r}} s_{r} !}$.

Definition 2 Let $w=y_{s_{1}} \ldots y_{s_{r}} \in Y^{*}$. For $N \geq k \geq 1$, the harmonic sum $\mathrm{A}_{w}(N ; k)$ is defined as $\mathrm{A}_{w}(N ; k)=\sum_{N \geq n_{1} \geq \ldots \geq n_{r} \geq k} \frac{1}{n_{1}^{s_{1}} \ldots n_{r}^{s_{r}}}$.

In particular, $\mathrm{A}_{y_{s_{1}} \ldots y_{s_{r}}}(N ; 1)$ stands for the multi-indexed notation $\mathrm{A}_{s_{1}, \ldots, s_{r}}(N)$.

For convenience, we will use the notation $\mathrm{A}_{w}(N)$ instead of $\mathrm{A}_{w}(N ; 1)$. We put $\mathrm{A}_{w}(0)=0$ and, for the empty word $\epsilon$, we put $\mathrm{A}_{\epsilon}(N)=1$, for any $N \geq 0$. The definition is extended to $\mathbb{Q}\langle Y\rangle$ by linearity.

Proposition 2 For any $u, v \in Y^{*}, \mathrm{~A}_{u} \sqsubset v(N ; k)=\mathrm{A}_{u}(N ; k) \mathrm{A}_{v}(N ; k)$.

(i) Note that the usual shuffle product and stuffle product of $u=y_{i} u^{\prime}$ and $v=y_{j} v^{\prime}$ are defined respectively by

$$
u \sqcup v=y_{i}\left(u^{\prime} \sqcup v\right)+y_{j}\left(u \sqcup v^{\prime}\right), \quad \text { and } \quad u \amalg v=y_{i}\left(u^{\prime} \leftarrow v\right)+y_{j}\left(u \leftarrow v^{\prime}\right)+y_{i+j}\left(u^{\prime} \leftarrow+v^{\prime}\right) .
$$


Proposition 3 For $w=y_{s} w^{\prime} \in Y^{*}$, we have $\mathrm{A}_{w}(N)=\sum_{N \geq l \geq 1} \frac{\mathrm{A}_{w^{\prime}}(l)}{l^{s}}$.

For $s_{1}>1, A_{w}(N)$ converges to a limit denoted by $\Theta(w)$ and the word $w$ is said to be convergent. By Formula $(5), \Theta(w)$ can be expressed as a linear combination of MZV.

More generally, as a consequence of the nature of coefficients occuring in the asymptotic expansion of $\mathrm{H}_{w}(N)$ [4], we get the following result

Proposition 4 Let $\mathcal{Z}$ be the $\mathbb{Q}$-algebra generated by $M Z V$, i.e. $\left\{\zeta(w), w \in Y^{*} \backslash y_{1} Y^{*}\right\}$ and let $\mathcal{Z}^{\prime}$ be the $\mathbb{Q}[\gamma]$-algebra generated by $\mathcal{Z}$. Then there exist algorithmically computable coefficients $b_{i} \in \mathcal{Z}^{\prime}, \kappa_{i} \in \mathbb{N}$ and $\eta_{i} \in \mathbb{Z}$ such that, for any $w \in Y^{*}$,

$$
\mathrm{A}_{w}(N) \sim \sum_{i=0}^{+\infty} b_{i} N^{\eta_{i}} \log ^{\kappa_{i}}(N), \text { for } \quad N \rightarrow+\infty
$$

\section{Asymptotic equivalent for $\operatorname{Var}\left(K_{n, d}\right)$}

In this section, we focus on the asymptotic equivalent of $\operatorname{Var}\left(K_{n, d}\right)$

$$
\operatorname{Var}\left(K_{n, d}\right) \sim\left(\frac{1}{(d-1) !}+\kappa_{d}\right) \ln ^{d-1}(n)
$$

$\kappa_{d}$ given by Formula (4). This one can be re-written, with our tools, in the following way:

$$
\kappa_{d}=\frac{1}{(d-1) !} \sum_{t=1}^{d-2}\left(\begin{array}{c}
d-1 \\
t
\end{array}\right) \sum_{l \geq 1} \frac{1}{l^{2}} A_{y_{1}^{t-1} \sqcup y_{1}^{d-2-t}}(l)
$$

Remind that we denote by $N_{2}(w)$ the number of occurences of the letter $y_{2}$ in $w$.

Theorem $1 \kappa_{d}=\frac{1}{(d-1) !} \sum_{w \in\left\{y_{1}, y_{2}\right\}_{d-3}}(-1)^{N_{2}(w)}\left(\begin{array}{c}2\left(d-2-N_{2}(w)\right) \\ d-2-N_{2}(w)\end{array}\right) \Theta\left(y_{2} w\right)$.

For example, for $d=7$, we get

$$
6 ! \kappa_{7}=\left(\begin{array}{c}
10 \\
5
\end{array}\right) \Theta(2,1,1,1,1)-\left(\begin{array}{l}
8 \\
4
\end{array}\right)(\Theta(2,2,1,1)+\Theta(2,1,2,1)+\Theta(2,1,1,2))+\left(\begin{array}{l}
6 \\
3
\end{array}\right) \Theta(2,2,2) .
$$

The last step consists in reducing into polyzêtas, and then use the reduction table. The following example make explicitly appear irreducible MZV of length $>1$, which was not observed before,

$$
\begin{aligned}
\kappa_{11} & =\frac{209}{302400} \zeta(5) \zeta(2) \zeta(3)+\frac{2893}{6048000} \zeta(2)^{2} \zeta(3)^{2}+\frac{3311}{460800} \zeta(3) \zeta(7) \\
& -\frac{517}{921600} \zeta(8,2)+\frac{39457}{9676800} \zeta(5)^{2}+\frac{426341}{221760000} \zeta(2)^{5}
\end{aligned}
$$

\section{Next terms in the Asymptotic Expansion}

Let us come back to Expression (1), that we can interpret, in terms of harmonic sums, this way

$$
\mathbb{E}\left(K_{n, d}^{2}\right)=\mathrm{A}_{y_{1}^{d-1}}(n)+\sum_{1 \leq t \leq d-1}\left(\begin{array}{l}
d \\
t
\end{array}\right) \sum_{l=1}^{n-1} \frac{1}{l} \mathrm{~A}_{y_{1}^{t-1}}(l) \mathrm{A}_{y_{1}^{d-t-1}}(l) \mathrm{A}_{y_{1}^{d-1}}(n ; l+1),
$$

Proposition 5 For any integers $n \geq l, \mathrm{~A}_{y_{1}^{d}}(n ; l)=\sum_{\substack{k_{1}+\ldots+d k_{d}=d \\ k_{1}, \ldots, k_{d}>0}} \frac{\mathrm{A}_{1}^{k_{1}}(n ; l) \ldots \mathrm{A}_{d}^{k_{d}}(n ; l)}{1^{k_{1}} k_{1} ! \ldots d^{k_{d}} k_{d} !}$

- Thanks to Proposition 2, we are able to turn each polynomial (in harmonic sums) into a linear combination of harmonic sums. 
- Finally, there are only sums over $l$ of type $\frac{A_{w}(l)}{l}$ left, but by Proposition 3 they simply reduce to $A_{y_{1} w}(n-1)$.

$$
\begin{aligned}
\operatorname{Var}\left(K_{n, 3}\right) & =\mathbb{E}\left(K_{n, 3}^{2}\right)-\mu_{n, 3}^{2} \\
& =\mathrm{A}_{1,1}(n)+3 \mathrm{~A}_{1}^{2}(n) \mathrm{A}_{1,1}(n-1)-12 \mathrm{~A}_{1}(n) \mathrm{A}_{1,1,1}(n-1) \\
& +6 \mathrm{~A}_{1}(n) \mathrm{A}_{1,2}(n-1)+18 \mathrm{~A}_{1,1,1,1}(n-1)-12 \mathrm{~A}_{1,1,2}(n-1) \\
& -12 \mathrm{~A}_{1,2,1}(n-1)+6 \mathrm{~A}_{1,3}(n-1)+3 \mathrm{~A}_{2}(n) \mathrm{A}_{1,1}(n-1)-\mathrm{A}_{1,1}^{2}(n) .
\end{aligned}
$$

- Using algorithms described in [4], we can now compute the asymptotic expansion of $\operatorname{Var}\left(K_{n, d}\right)$.

Theorem 2 There exist algorithmically computable coefficients $\alpha_{i}, \beta_{j, k} \in \mathcal{Z}^{\prime}$ such that, for any dimension $d$ and any order $M$,

$$
\operatorname{Var}\left(K_{n, d}\right)=\sum_{i=0}^{d-1} \alpha_{i} \ln ^{i}(n)+\sum_{j=1}^{M} \frac{1}{n^{j}} \sum_{k=0}^{2 d-2} \beta_{j, k} \ln ^{k}(n)+\mathrm{o}\left(\frac{1}{n^{M}}\right) .
$$

For example,

$$
\begin{aligned}
\operatorname{Var}\left(K_{n, 3}\right) & =\left(\frac{1}{2}+\kappa_{3}\right) \ln ^{2}(n)+(-10 \zeta(3)+2 \zeta(2) \gamma+\gamma) \ln (n)+\frac{1}{2} \gamma^{2} \\
& -10 \zeta(3) \gamma+\frac{83}{10} \zeta(2)^{2}+\zeta(2) \gamma^{2}+\frac{1}{2} \zeta(2)+\mathrm{o}(1)
\end{aligned}
$$

\section{References}

[1] Z.D. Bai, C.C. Chao, H.K. Hwang, W.Q. Liang.- On the variance of the number of maxima in random vectors and its applications, Annals of Applied Probability, 8(3), pp. 886-895, 1998. MathReview: 99f:60019

[2] Z.D. Bai, L. Devroye, H.K. Hwang, T.H. Tsai.- Maxima in hypercubes, Random Structures and Algorithms, 27 (3), pp. 290-309, 2005.

[3] O. Barndorff-Nielsen, M. Sobel.- On the distribution of the number of admissible points in a vector random sample, Theory Proba. Appl., 11, pp.249-269, 1966.

[4] C. Costermans, J.Y. Enjalbert, Hoang Ngoc Minh.-Algorithmic and combinatoric aspects of multiple harmonic sums, in the proceedings of Aofa, Barcelone, 06-10 June, 2005.

[5] V. M. Ivanin.- Calculation of the dispersion of the number of elements of the Pareto set for the choice of independent vectors with independent components, Theory of Optimal Decisions, Akad. Nauk. Ukrain. SSR Inst. Kibernet., Kiev., pp. 90-100, 1976. 